\title{
Contribuições do trabalho de campo para aulas de geografia no ensino fundamental
} Contributions of fieldwork to geography classes in elementary school

Contribuciones del trabajo de campo a las clases de geografía en la escuela primaria

Recebido: 18/04/2020 | Revisado: 22/04/2020 | Aceito: 07/05/2020 | Publicado: 16/05/2020

\section{Igo Marinho Serafim Borges}

ORCID: https://orcid.org/0000-0002-3662-1859

Universidade Estadual da Paraíba, Brasil

E-mail: igomarinho27@gmail.com

Raphael Lucas Jacinto Almeida

ORCID: https://orcid.org/0000-0001-7232-2373 Universidade Federal do Rio Grande do Norte, Brasil

E-mail: raphaelqindustrial@gmail.com

Carla Andreza Oliveira Lima

ORCID: https://orcid.org/0000-0003-2323-7345

Universidade Estadual da Paraíba, Brasil

E-mail: c.andreza@outlook.com

Amanda Cristiane Gonçalves Fernandes

ORCID: https://orcid.org/.0000-0001-8462-6171

Universidade Estadual da Paraíba, Brasil

E-mail: amandafernandestt@gmail.com

Ramon Marinho Gomes

ORCID: https://orcid.org/0000-0001-8382-8628

Universidade Estadual da Paraíba, Brasil

E-mail: ramongomes032@gmail.com

Welitemara da Silva Araújo

ORCID: https://orcid.org/0000-0002-9594-9109

Universidade Federal do Pará, Brasil

E-mail:wellyaraujojb05@gmail.com 
Karine Rodrigues de Andrade

ORCID: https://orcid.org/0000-0002-3809-9267

Universidade Estadual da Paraíba, Brasil

E-mail: karineandrade08@gmail.com

Maria Lucilane de Andrade Silva

ORCID: https://orcid.org/0000-0002-2216-2700

Universidade Estadual da Paraíba, Brasil

E-mail: Marialucyandrade1@gmail.com

Thiago da Silva Lima

ORCID: https://orcid.org/0000-0002-3364-3980

Universidade Estadual da Paraíba, Brasil

E-mail: thiagosilva.geo@bol.com.br

Emanoel Cavalcante Silva

ORCID: https://orcid.org/0000-0002-9239-7396

Universidade Estadual da Paraíba, Brasil

E-mail: netinhocavalcante2008@ hotmail.com

\section{Resumo}

Este trabalho tem como objetivo discutir a importância do trabalho de campo como recurso metodológico no contexto do processo de ensino e aprendizagem e nas aulas de Geografia. De modo a colocar em prática os conhecimentos adquiridos em sala de aula, utilizou-se como metodologia trabalhos de campo com alunos do $7^{\circ}$ ano (B), da Escola de Ensino Fundamental Judith Barbosa de Paula Rego, localizada na cidade de Queimadas-PB. Os resultados demonstram que as aulas teóricas acompanhadas das aulas práticas em campo proporcionam um maior desempenho no aprendizado. Houve aumento nas notas, onde foi possível observar que a partir dos trabalhos de campo os alunos tiveram maior interesse em estudar as ciências geográficas, saindo do abstrato para o experienciado tiveram a oportunidade de associar o que já conheciam a partir de informações do cotidiano deles com os conteúdos da Geografia vistos em sala de aula. O desenvolvimento de trabalhos com a aula de campo possibilita a articulação entre teoria e prática, bem como forma de agregar os saberes dos alunos com o conhecimento do livro didático, o que é de suma importância no processo de ensinoaprendizagem.

Palavras-chave: Praticas em campo; Ensino e aprendizagem; Ensino de Geografia; Trabalho de Campo. 


\section{Abstract}

This paper aims to discuss the importance of fieldwork as a methodological resource in the context of the teaching and learning process and in Geography classes. In order to put into practice the knowledge acquired in the classroom, fieldwork with 7th grade students (B), from the Judith Barbosa de Paula Rego Elementary School, located in the city of Queimadas-PB, was used as methodology. The results demonstrate that the theoretical classes accompanied by practical classes in the field provide a greater performance in learning. There was an increase in grades, where it was possible to observe that from the fieldwork students had a greater interest in studying the geographic sciences, leaving the abstract to the experienced they had the opportunity to associate what they already knew from their daily information with the contents of Geography seen in the classroom. The development of works with the field class allows the articulation between theory and practice, as well as a way to aggregate the students' knowledge with the knowledge of the textbook, which is extremely important in the teaching-learning process.

Keywords: Field practices; Teaching and learning; Geography teaching; Fieldwork.

\section{Resumen}

Este artículo tiene como objetivo discutir la importancia del trabajo de campo como recurso metodológico en el contexto del proceso de enseñanza y aprendizaje y en las clases de Geografía. Para poner en práctica los conocimientos adquiridos en el aula, se utilizó como metodología el trabajo de campo con estudiantes de $7^{\circ}$ grado (B), de la Escuela Primaria Judith Barbosa de Paula Rego, ubicada en la ciudad de Queimadas-PB. Los resultados demuestran que las clases teóricas acompañadas de clases prácticas en el campo proporcionan un mayor rendimiento en el aprendizaje. Hubo un aumento en las calificaciones, donde fue posible observar que, desde el trabajo de campo, los estudiantes tenían un mayor interés en estudiar las ciencias geográficas, dejando el resumen a los experimentados que tenían la oportunidad de asociar lo que ya sabían de su información diaria con Los contenidos de Geografía vistos en el aula. El desarrollo de trabajos con la clase de campo permite la articulación entre la teoría y la práctica, así como una forma de agregar el conocimiento de los estudiantes con el conocimiento del libro de texto, que es extremadamente importante en el proceso de enseñanza-aprendizaje.

Palabras clave: Prácticas de campo; Enseñanza y aprendizaje; Enseñanza de geografía;

Trabajo de campo. 


\section{Introdução}

Ensinar geografia na atualidade, assim como todas as outras ciências ensinar não é algo fácil. $\mathrm{Na}$ contemporaneidade torna-se difícil impressionar os alunos com atividades tecnológicas, visto que, os mesmo são nativos digitais, crianças que já nasceram no mundo digital. Desta forma, busca-se outras formas metodológicas para que os alunos tenham interesse em aprender as ciências geográficas e um delas é a de laboratórios de campo.

Borges (2019) afirma que é de extrema necessidade que o docente busque meios para deixar sua aula mais atrativa e desperte o interesse desses alunos, que por alguma razão estão desmotivados.

Com base nisso, este trabalho busca enaltecer as atividades extra sala de aula como é caso do trabalho de campo. A utilização desse recurso proporciona atratividade nas aulas de geografia, desta forma fugindo da rotina de atividades da sala de aula e fazendo com que o teoria e prática caminhem juntos facilitando o processo de ensino na atualidade.

Krasilchik (2004) afirma que um professor pode expor os conteúdos por meio de uma aula expositiva, o que pode ser uma experiência informativa, divertida e estimulante, dependendo da forma como ocorra o preparo da aula. Porém em alguns casos, é cansativa e pouco contribui para a formação dos alunos. Uma saída da escola ou trabalho de campo, também chamadas de visitas, passeios e excursões podem estar inseridos no currículo escolar. Esta atividade é caracterizada por ser mais flexível, por trabalhar o conteúdo proposto e acontecer em ambiente extraclasse da instituição educacional (Krasilchik, 2004; Morais \& Paiva, 2009).

Nesse contexto deixar de lado o ensino da Geografia tradicional, onde os trabalho de campo eram voltados para a observação e definição dos elementos contidos nas paisagens, resultando numa prática de ensino meramente descritiva do espaço geográfico. Nesse contexto, estudar Geografia reduzia-se apenas a ter ou desenvolver habilidade "fotográfica", capazes de guardar inúmeras imagens do espaço estudado e suas respectivas informações, construindo assim o famoso "decoreba" já que o objetivo principal era reduzido à descrição dos diferentes lugares da superfície terrestre, ou seja, decorar nomes de países, estados e cidades, atribuindo a cada lugar um tipo de clima, entre outros.

Contudo, não implica dizer que o trabalho de campo não deve se basear também na observação, ao contrário, a observação é primordial para um estudo sobre os elementos da paisagem, aguçando um senso crítico e investigador sobre o espaço, mas não deve se restringir a isso, assim afirma Braun (2007): “o trabalho de campo significa observar. Permite 
tanto a observação íntima de certos aspectos de comportamento do espaço, como descrevê-los de forma útil". Nesse sentido, é preciso entender que o alunado está naquele momento relacionando a teoria apreendida em sala de aula com a prática, construindo, por meio da observação e análise, posicionamentos críticos e sociais.

Para Alentejano (2017) O trabalho de campo não pode ser mero exercício de observação da paisagem, mas partir desta para compreender a dinâmica do espaço geográfico, num processo mediado pelos conceitos geográficos. Fazer trabalho de campo representa, portanto, um momento do processo de produção do conhecimento que não pode prescindir da teoria, sob pena de tornar-se vazio de conteúdo, incapaz de contribuir para revelar a essência dos fenômenos geográficos (Ibid, 2017).

Diante do exposto, é considerável frisar que para que a aula de campo de fato promova o desenvolvimento da aprendizagem de maneira satisfatória, o docente precisa distanciar-se do "vou fazer porque tenho que fazer", é preciso que seja planejado todo desenvolvimento do trabalho de campo, exigindo um processo em que segue etapas, as quais são: planejamento, execução, análises e relatórios, situando constantemente a atividade com os objetivos que norteiam a aula.

Mediante o exposto, o presente trabalho objetiva relatar a pratica da aula de campo como aporte para o aprimoramento das aulas de Geografia no ensino básico. Desta forma, buscando unir teorias a pratica, relacionando os conteúdos visto em sala com a realidade dos alunos.

\section{Materiais e Métodos}

Uma pesquisa é feita para trazer novos saberes para a sociedade como preconiza Pereira et al. (2018). A presente pesquisa tem modalidade interventiva com características qualitativas. O estudo foi realizado em sala de aula com os alunos por meio de aulas dialogadas e debatidas, além de leitura e utilização de Datashow. Essas aulas tiveram como temas em destaque: os processos migratórios, densidade demográfica, aspectos naturais e aspectos da economia do nordeste brasileiro. Foi possível, a partir da participação dos alunos e das suas percepções um debate produtivo sobre as temáticas abordadas na aula de campo.

Assim sendo, está pesquisa foi baseada no processo investigativo, de cunho colaborativo na qual foi utilizada uma abordagem qualitativa que favorece a compreensão do assunto a partir da investigação realizada com os participantes (Lüdke \& André, 1993). 
Research, Society and Development, v. 9, n. 7, e341973762, 2020

(CC BY 4.0) | ISSN 2525-3409 | DOI: http://dx.doi.org/10.33448/rsd-v9i7.3762

Desta forma, evidenciar e validar a importância de aulas de campo no ensino de geografia na rede pública de ensino fundamental. Partimos, portando, da reflexão do trabalho de campo realizado na turma do $7^{\circ}$ ano "B" da Escola de Ensino Fundamental Judith Barbosa de Paula Rego, em Queimadas-PB (Figura 1).

Figura 1. Localização da área do estudo.

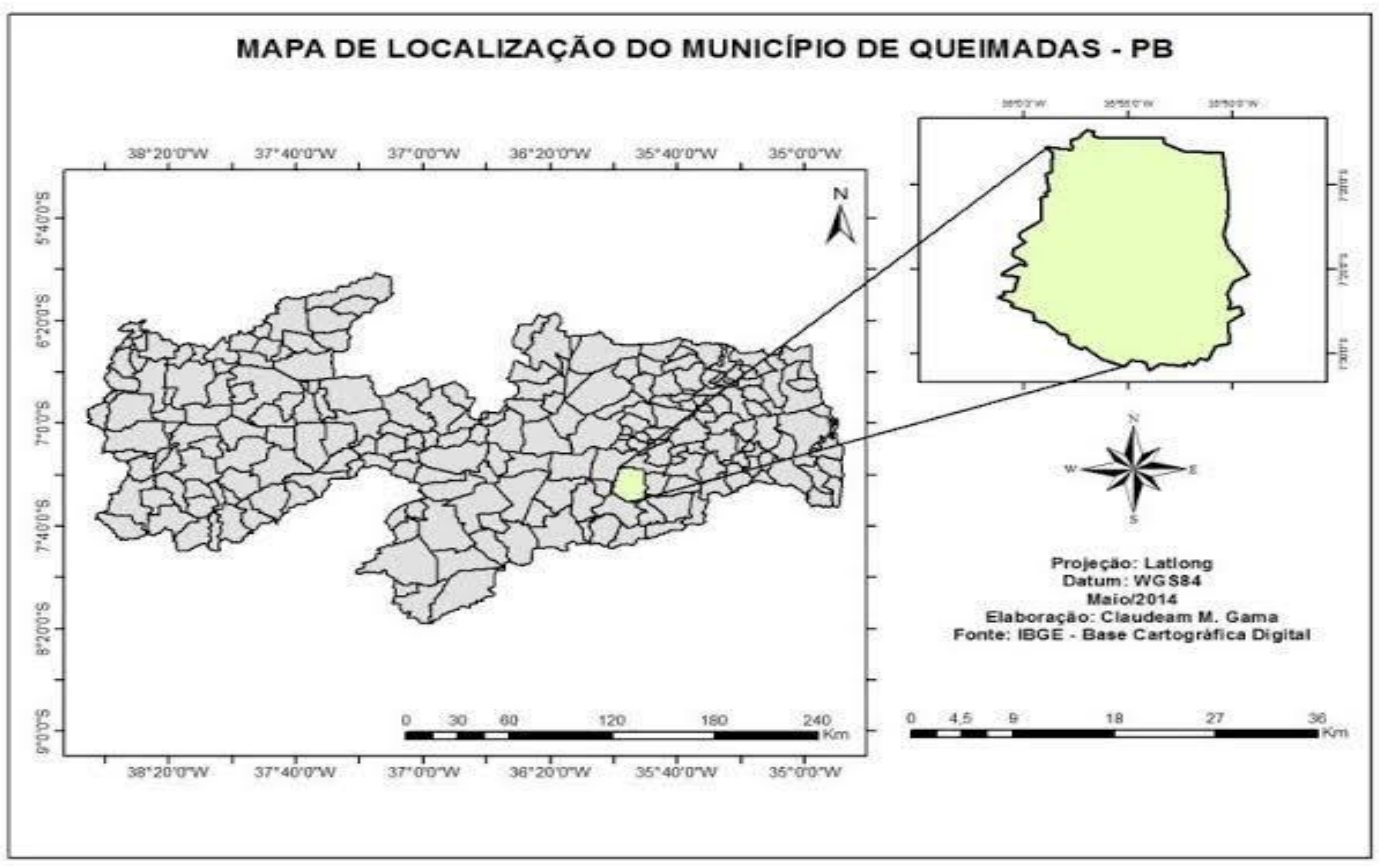

Fonte: Gama, 2015.

As aulas de campo foram desenvolvidas em dois momentos: $\mathrm{O}$ primeiro na cidade, a qual a instituição está localizada. E o segundo realizado no ponto turístico da cidade de Fagundes-PB, Pedra de Santo Antônio. (Figura 2). 
Figura 2. Localização da área do estudo.

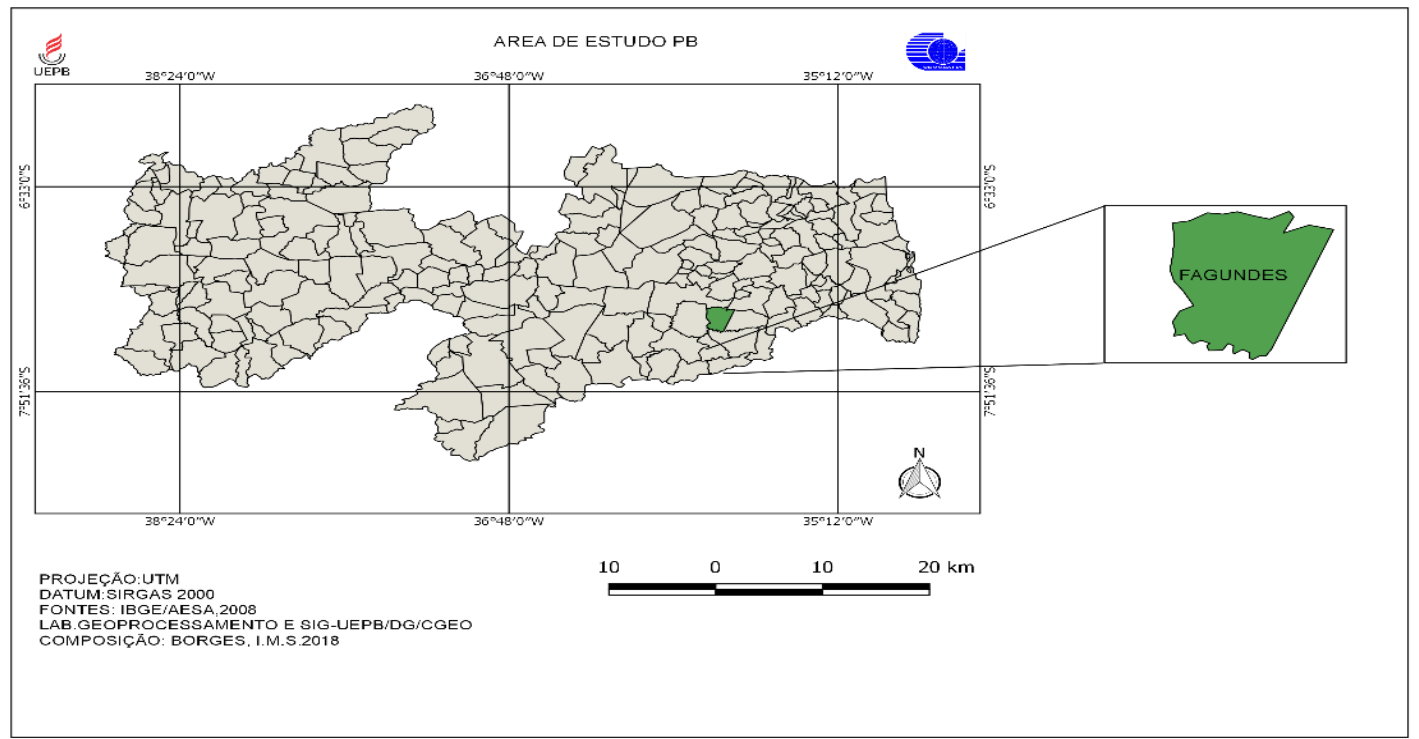

Fonte: Borges, 2018.

Nas aulas foram desenvolvidas temáticas abordadas em classe como é o caso de movimentos migratórios, vegetação, hidrografia e relevo da região nordeste, pais e cidade populosos entre outros assuntos.

Um dos principais métodos aplicadas de início foi a de debates e participações dos alunos visto que, suas visões, críticas e analises são fundamental para as discussões, ou seja, fazendo uma inter-relação entre as colaborações presente no contexto professor/aluno. Um dos principais objetivos era unir teoria à prática e colaborar com o desenvolvimento do ensino-aprendizagem.

A percepção do meio, como preconiza Menezes (2011), torna-se de grande importância para que possamos compreender melhor as inter-relações entre o homem e o ambiente, podendo surgir com isso uma necessidade de preservação com as visitas a campo e assim criar uma maior consciência ambiental entre os alunos, ampliando o conhecimento, as perspectivas e aspirações acerca do tema proposto.

\section{Resultados e Discussão}

A partir do desenvolvimento das aulas em geral, pode-se notar uma melhor compreensão acerca das tematicas trabalhadas. Melhor desempenho nas atividades, melhor desempenho nas notas obtidas em atividades avaliativas, maior mobilidade de discussão das 
tematicas trabalhadas em sala de aula. A exemplo, o olhar dos alunos a partir da aula de campo que se desenvolveu na zona urbana do município de Queimadas-PB, tendo dentre as temáticas abordadas o processo de urbanização, no qual os alunos relataram e demonstrarm os conhecimentos anteriormente trabalhados em sala de aula, tais como: processo de verticalização, a composição da inflaestrutura municípal, êxodo rural, a influência do comércio no desenvolvimento das cidades, a segregação sócio-espacial, entre outros.

Gerando desta forma, um debate mais sólido visto que, se tratava do lugar onde eles vivem. Elucidando e destacando o conhecimento empirico e unindo-o as discussões trabalhadas em aula. Buscando, unior a teoria à prática. Não tendo o livro didático como único aporte teórico.

Logo após, foi desenvolvido outra aula de campo no município de Fagundes tendo mais uma vez o objetivo unir teoria a prática. A proxima aula de campo foi realizada na pedra de Santo Antônio na cidade de Fagundes-PB. Os alunos poderam interagir mais com os conteudos e colocar em prática a teoria aplicada em sala, diversas tematicas foram abordadas como: turismo e fatores economicos, conservaçao e preservação ambiental, diversos tipos de solo, fatores climático e meteórologicos.

Fazer com que teoria/prática caminem juntas, desta froma, possibilitando aos alunos enxergar as ciências geográficas de outra forma. Fazendo com que o livro didático seja um suporte para o ensino e não o único instrumento de ensino-aprendizagem.

Como propostas de atividades para os alunos tiveram presentes elaboracao de relatórios, atividade de revisão do conteúdo trabalho em sala e nas aulas de campo. Elaboracao de um concurso de fotografia e defesa de porque suas fotos deveriam ser ser as vencedoras onde os alunos poderam apontar situacoes presentes no seu dia-a-dia vistas no laboratorio de campo.

Entre essas discussões, destaca-se David Ausubel (1918-2008) que desenvolveu a teoria da aprendizagem significativa. Esta enfatiza que o conhecimento só passa a ser significativo para o aluno à medida que a nova informação se liga àquilo que o aluno já sabe, ou seja, os chamados conceitos prévios (Ribeiro e Nuñez, 2004).

Moreira (2011) define esses conceitos prévios como subsunções ou ideia-âncora e, descreve a aprendizagem significativa como sendo: aquela em que ideias expressas simbolicamente interagem de maneira substantiva e não-arbitrária com aquilo que o aprendiz já sabe. Substantiva quer dizer não literal, não ao pé da letra, e não arbitrária significa que a interação não é com qualquer ideia prévia, mas sim com algum conhecimento especificamente relevante já existente na estrutura cognitiva do sujeito que aprende. 
As práticas das aulas de campo despertam nos nossos alunos uma visão inovadora sobre o que é as ciências geográficas dentro de tantos conceitos e definições da ciência, o trabalho metodológico com aula de campo torna o ensino bem mais inovador. O uso do lúdico como proposta de ensino faz com que os alunos consigam interagir mais com as aulas e desta forma desenvolver seus próprios conceitos, proporcionando um maior desenvolvimento das suas capacidades de aprendizagem.

\section{Considerações Finais}

Uma das dificuldades encontradas na ciência geográfica e que perpassa por décadas dando a impressão que não houve uma grande evolução no ensino, é esse status de ciência desinteressante e decorativa que traz definições prontas, onde muitas vezes não é apresentado aos alunos o processo de como ocorreram os fatos e por quê ocorreram. A aula de campo proporciona o contato com o objeto de estudo possibilitando uma maior compreensão e associação do conteúdo. Trará uma nova forma de ver a Geografia, de entende-la, enfatizando que essa disciplina não se trata de dar nomes as coisas, mas de enteder como elas ocorreram e entender a dinâmica entre sociedade/natureza.

A aula de campo possibilita uma experiência com detalhes, desperta a curiosidade. $\mathrm{O}$ observar, o tocar, o sentir, fazem parte do processo de ensino, corroborando para que os alunos entendem as influências existentes entre a natureza e entre esta e a sociedade.As práticas de campo proporcionam aos alunos uma grande aproximação com os conteúdos vistos em sala de aula, além disso as aulas práticas podem articular uma sequência interdisciplinar entre as diversas ciências. Portanto, trabalhar com os alunos para além da sala de aula é de extrema necessidade visto que possibilita um boa formação crítica e alinhada as realidades presentes no cotidiano, interligando os alunos aos fatores sociais que os envolvem.

Como sugestão para trabalhos futuros, podemos fazer a discussão sobre o desenvolvimento de alunos do ensino fundamental com metodologia ativa, fazer também trabalhos relacionados ao uso de tecnologias no ensino fundamental e médio, uma proposta lúdica no ensino de geografia.

\section{Referências}

Alentejano, PRR \& Rocha-Leão, OM. (2017). Trabalho de campo: uma ferramenta essencial para os geógrafos ou um instrumento banalizado? Boletim Paulista de Geografia, (84), 51-68. 
Ausubel, DP. (1982). Teoria da Aprendizagem signiticativa. Rio de Janeiro: Interamericana.

Borges, IMS. (2019). As contribuições do trabalho de campo para o processo de ensinoaprendizagem em geografia. Anais do Encontro de Iniciação à Docência, VII ENID Campina Grande, PB, Brasil.

Braun, AM. (2007). Rompendo os muros da sala de aula: o trabalho de campo na aprendizagem de geografia. Ágora, 13(1), 250-272.

Krasilchik, M. Prática de Ensino de Biologia. 4.ed., São Paulo: EDUSP, 2004.

Lüdke, M \& André, MEDA. (1993). Combinando pesquisa e prática no trabalho e na formação de professores. Ande, 12, 31-37.

Menezes, JPC. (2011). Percepção Ambiental dos visitantes do Parque Municipal Bosque JohnKennedy - Araguari, MG. Revista eletrônica do Mestrado em Educação Ambiental, 26.

Moreira, MA. (2011). Aprendizagem significativa: a teoria e textos complementares. São Paulo: Editora Livraria da Física, 1ed. 179p.

Pereira, AS, Shtsuka, DM, Parreira, FJ \& Shitsuka, R. (2018). Metodologia da pesquisa científica. [e-book]. Santa Maria. Ed. UAB/NTE/UFSM. Acesso em: 14 maio 2020. Disponível em: https://repositorio.ufsm.br/bitstream/handle/1/15824/Lic_Computacao_MetodologiaPesquisa-Cientifica.pdf?sequence $=1$.

Ribeiro, RP \& Nunez, IB. (2004). A aprendizagem significativa e o ensino de Ciências Naturais. Fundamentos do ensino-aprendizagem das ciências naturais e da matemática: o novo ensino médio. Porto Alegre: Sulina, 29-42.

Silva, EM \& Melo, A. (2008). Estudo preliminar sobre trabalhos de campo na perspectiva da educação inclusiva: um desafio para os futuros professores de Geografia. Caminhos de Geografia, 9(25). 
Research, Society and Development, v. 9, n. 7, e341973762, 2020

(CC BY 4.0) | ISSN 2525-3409 | DOI: http://dx.doi.org/10.33448/rsd-v9i7.3762

Porcentagem de contribuição de cada autor no manuscrito

Igo Marinho Serafim Borges - 10\%

Raphael Lucas Jacinto Almeida - 10\%

Carla Andreza Oliveira Lima - 10\%

Amanda Cristiane Gonçalves Fernandes - 10\%

Ramon Marinho Gomes - 10\%

Welitemara da Silva Araújo - 10\%

Karine Rodrigues de Andrade - 10\%

Maria Lucilane de Andrade Silva - 10\%

Thiago da Silva Lima- $10 \%$

Emanoel Cavalcante Silva - 10\% 